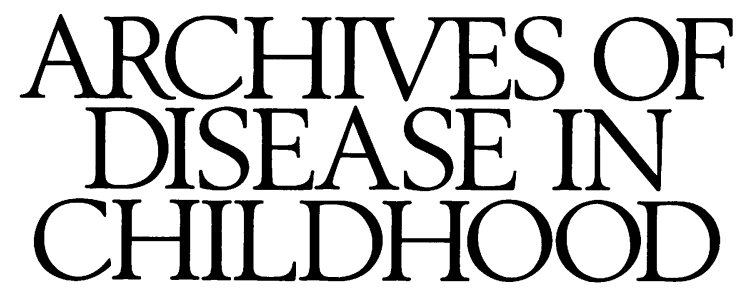

The fournal of the British Paediatric Association

\title{
The use of plasma in the neonatal period
}

For many years the use of plasma and plasma expanders has been common in neonatal nurseries and they have taken a place in the armamentarium of therapies available to the neonatologist. There has been little scientific testing of their efficacy, however, despite this wide use in clinical practice at not inconsiderable cost.

The substances do have many admirable qualities: they contain protein, expand the circulating volume, and fresh frozen plasma contains clotting factors. As such these substances are attractive but on the other hand there are disadvantages-for example, they may produce circulatory overload and can transmit infection. It is therefore surprising that so little research has been performed on the use of these substances in the neonatal period.

\section{Use of plasma}

\section{INTRAVENTRICULAR HAEMORRHAGE}

It has been suggested for many years that defects of haemotasis were associated with intraventricular haemorrhage but correction of such defects resulted in equivocal conclusions. In a trial of the use of fresh frozen plasma with ultrasonic diagnosis of intraventricular haemorrhage, however, Bevereley et al showed that the incidence of intraventricular haemorrhage was reduced by fresh frozen plasma given to babies on admission to the neonatal unit and again at 24 hours of age. ${ }^{1}$ Significant intraventricular haemorrhages grades III and IV occurred in eight of 37 control babies compared with three of 36 treated babies. This study also showed that the difference was not related to blood clotting deficiencies and the authors conclude that the results might be due to improvement of vascular stability. Such studies are interesting and should be repeated together with long term follow up. Further, comparison should be made between fresh frozen plasma and other plasma expanding agents.

\section{RESUSCITATION}

Many infants are born asphyxiated or hypotensive and on occasions this is due to fetal haemorrhage. In this situation it is reasonable to expand the circulating volume by means of plasma. Whereas the use of whole blood transfusion would be preferable, this is not always available in labour wards but plasma or albumin is. Again, formal studies appear not to be available but despite this deficiency, such use of these fluids is recommended in most neonatal textbooks.

\section{COAGULATION DEFECTS}

Turner and his colleagues noted the abnormal coagulation profiles of sick newborn infants and set out to correct such defects with haematological success but with clinical failure. ${ }^{2}$ This result was interesting but not surprising, as what was being done was to apply a fire extinguisher to a fire rather than to stop the fuel supply. Coagulation defects occur in the most gravely ill babies and are a consequence of rather than the cause of the clinical condition, improvement of which is of paramount importance.

\section{POLYCYTHAEMIA}

It was demonstrated by Stone et al that erythrocytes had a major effect on blood viscosity. ${ }^{3}$ The effect of polycythaemia (packed cell volume of venous blood $>0.7$ ) is to increase the possibility of intravascular thrombosis. It is therefore mandatory to reduce whole blood viscosity at such levels or if the baby develops symptoms suggestive of hyperviscosity such as jitteriness or irritability. ${ }^{4}$ This is usually easily done by exchanging whole blood for plasma in a volume of $10-20 \mathrm{ml} / \mathrm{kg}$ body weight. This means of treatment successfully reduces the packed cell volume and whole blood viscosity.

\section{DISADVANTAGES OF PLASMA}

There are two major problems in the use of plasma in the neonatal period: that of cost and of the risk of transmission of infection. The principle hazard, at least in America, in the use of fresh frozen plasma would appear to be the transmission of non-A, non-B hepatitis with the reported incidence in adults of 3 to $10 \%, 5$ and there is some risk of transference of cytomegalovirus infection. There are no published reports of this in the neonatal period but certainly such risks may exist.

\section{Use of albumin}

Albumin is used primarily for two purposes: for volume expansion and to bind bilirubin. Volume expansion is needed in a variety of clinical situations that induce shock and most delivery wards and neonatal units store salt poor albumin for infusion. The logic of giving albumin (10-20 $\mathrm{ml} / \mathrm{kg}$ body weight) in this situation is that colloid is being infused, it will stay in the vascular space, and will maintain or improve the blood pressure. Disadvantages have been suggested for many years-in particular volume overload and an increased likelihood of patent ductus arteriosus. In clinical practice, however, if the correct dose is given, these complications are seldom seen. In any event it seems logical 
that treatment of the presenting life threatening event takes precedence over such disadvantages.

Albumin is also used before exchange transfusion to enhance bilirubin removal but the evidence for this is debated. ${ }^{6}$ This means of treatment was employed extensively in the era of $\mathrm{Rh}$ haemolytic disease but is now much less often used.

\section{RESPIRATORY DISTRESS SYNDROME}

The facts that infants with respiratory distress syndrome are oedematous and have low serum albumin concentrations led to the idea that replacement may confer benefits. Greenough et al most recently expressed this possibility and showed that infusions caused a diuresis and weight loss in sick infants. ${ }^{7}$ However, such studies really only support the contention that infants with respiratory distress syndrome are hypovolaemic and therefore suffer from underperfusion. What is unclear is whether this matters or not. Perhaps a more interesting line of study would be to look at the effects of plasma expansion early in postnatal life-there were studies in the late 1960s suggesting that umbilical cord oedema and serum albumin concentrations could be used as markers for the development of respiratory distress syndrome. What would happen now if we were to expand the circulating volume in the first few minutes of extrauterine life and then follow up with modern intensive care methods? It is unlikely that albumin will be curative but it might shorten or reduce the requirement for intensive care.

\section{Conclusion}

The use of these substances in neonatal care is widespread but poorly studied. This is unfortunate as any means of treatment must be formally assessed to ensure that it is properly used and maximum benefit obtained. Formal studies should be undertaken in order to achieve this goal.

The Nuffield Department of Child Health,

G MCCLURE

The Queen's University of Belfast,

Institute of Clinical Science,

Grosvenor Road,

Belfast BT12 6Bf,

Northern Ireland

1 Beverley DW, Pitts-Tucker TJ, Congdon PJ, Arthur RJ, Tate G. Prevention of intraventricular haemorrhage by fresh frozen plasma. Arch Dis Child 1985;60:710-3.

2 Turner T, Prowse CV, Prescott RJ, Cash JD. A clinical trial on the early detection and correction of haematocrit defects in selected high-risk neonates. Br f Haematol 1981;47:65-75.

3 Stone HO, Thompson HK Jr, Schmidt-Lielson K. Influence of erythrocyte on blood viscosity. Am $\mathcal{F}$ Physiol 1968;214:913.

4 Halliday HL, McClure G, Reid MMcC. Handbook of neonatal intensive care. 3rd Ed. London: Baillière Tindall, 1989.

5 Bove JR. Transfusion-transmitted diseases: current problems and challenges. Prog Hematol 1986;XIV:123-47.

6 Chan G, Schiff D. Variance in albumin loading in exchange transfusions. f Pediatr 1976;88:609.

7 Greenough A, Greenall F, Gamsu HR. Immediate effects of albumin infusion in ill premature neonates. Arch Dis Child 1988;63:307-9.

\section{Is routine endotracheal suction justified?}

New developments provide the opportunity to answer this and other questions quickly and inexpensively.

The precise role of routine endotracheal suction in intubated babies is controversial. Tracheobronchial suction can reduce respiratory system resistance ${ }^{1}$ and should be performed whenever secretions accumulate, to prevent airway obstruction. However there are many potential hazards, including atelectasis, ${ }^{2}$ bradycardia, ${ }^{34}$ decreased lung compliance, ${ }^{4}$ hypoxia, ${ }^{4}$ transient increases in arterial and intracranial pressure and cerebral blood flow velocity, ${ }^{46}$ bacteraemia, ${ }^{7}$ and pneumothorax. ${ }^{8-10}$ Although they are less noticeable, fluctuations in physiological variables due to suction still occur after paralysis ${ }^{11}$ or when disconnection from the ventilator is avoided by using a side port adaptor. $^{12} 13$ Some of these hazards, for example pneumothorax, may be particularly dangerous in very preterm infants within the first three days of life, ${ }^{14}$ a period when secretions are usually scanty. Because of the risks, some workers question whether routine endotracheal suction is justified. ${ }^{46}$ Nevertheless, in many neonatal units endotracheal suction at intervals of one to six hours is still a routine procedure for all intubated babies, regardless of age or gestation. We remain collectively uncertain not only whether routine endotracheal suction is justified, but how it should be done. Sometimes the baby is 'preoxygenated', sometimes not. Usually suction is preceded by instillation of $0.25-0.5 \mathrm{ml}$ normal saline but occasionally as much as 2.0 $\mathrm{ml}$ is used. ${ }^{10}$ Sometimes the baby gets chest physiotherapy, which can increase the weight of secretions obtained ${ }^{15}$ but may exacerbate hypoxia.

Endotracheal suction may be needed more often when inspired gas is poorly humidified, because this inhibits mucociliary clearance and thickens tracheobronchial secretions. In a non-randomised study, Lomholt et al reported a tenfold increase in the risk of plugging of the endotracheal tube with viscid secretions when inspired gas was less than
$70 \%$ saturated at $37^{\circ} \mathrm{C}$, which is equivalent to an absolute humidity of $31 \mathrm{mg} \mathrm{H} \mathrm{H}_{2} \mathrm{O} / \mathrm{l}^{16}$ It might thus be sensible to arrange for the hospital medical physics department to check periodically that intubated babies do not breathe gas of lower humidity than this, because inspired gas humidity varies widely during routine clinical practice (MFD O'Hagan et $a l$, unpublished observations).

For the jobbing neonatologist, these issues raise important practical questions. Is it better to have a policy of suction only when impending airway obstruction is suspected than a policy of routine suction? If so-for which babies? Should endotracheal suction be preceded by chest physiotherapy?

These questions are most likely to be answered successfully in a large randomised controlled trial in which infants within each centre are randomly assigned to different policies. This would ensure that variations in case mix or clinical practice, which are inevitable, were balanced evenly between centres. To allow more than one question to be answered simultaneously a factorial design could be used so that infants were randomly assigned to either (a) routine endotracheal suction or (b) suction only when impending airway obstruction was suspected and, after the first 72 hours of life, to either (c) routine physiotherapy or (d) physiotherapy only when specifically indicated. Essential measures of outcome might include pneumothorax, duration of treatment with supplementary oxygen and with artificial ventilation, length of hospital stay, and abnormal ultrasound appearances at 6 weeks of age. It would be realistic to expect only a moderate reduction in the rate of any single adverse outcome, for example from $20 \%$ to $15 \%$. A study would have a good chance ( $80 \%$ power) of showing such a reduction at a conventional level of significance $(2 p=0.05)$ if it recruited a total of 2000 infants.

Now that paediatricians, nurses, epidemiologists, and parents are collaborating on an unprecedented scale to investigate the role of surfactant in the OSIRIS and CURO- 